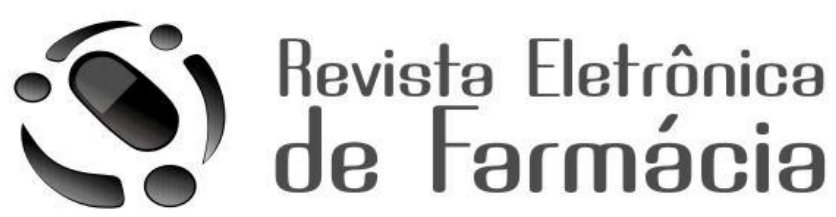

REF - ISSN 1808-0804 Vol. X (2), 43 - 57, 2013.

\title{
VALIDAÇÃO DE METODOLOGIA ANALÍTICA PARA DOSEAMENTO E ESTUDO DA EQUIVALÊNCIA FARMACÊUTICA DE COMPRIMIDOS DE SINVASTATINA 20 MG
}

METHODOLOGY VALIDATION FOR ASSAY AND PHARMACEUTICAL EQUIVALENCY STUDY FOR SIMVASTATIN TABLETS 20 MG

VALIDACIÓN DE MÉTODOS DE ANÁLISIS PARA LA DETERMINACIÓN Y ESTUDIO DE EQUIVALENCIA FARMACÉUTICA DE COMPRIMIDOS SIMVASTATINA 20 MG

\footnotetext{
${ }^{1 *}$ Karine Modolon Zepon, ${ }^{2}$ Gisleine Fratoni, ${ }^{1}$ Larissa Sakis Bernardi, ${ }^{1}$ Karina Valerim Teixeira Remor

${ }^{1}$ Docente do curso de Farmácia - Universidade do Sul de Santa Catarina.

${ }^{2}$ Discente do curso de Farmácia - Universidade do Sul de Santa Catarina.

*autor para correspondência: karine@cimject.ufsc.br
}

Recebido em 11/07/2012, Aceito em 27/06/2013

\section{RESUMO}

Estatinas, como a sinvastatina, agem na diminuição dos níveis séricos de colesterol total e de lipoproteína de baixa densidade. Com a expiração da patente da sinvastatina, novas marcas sob as formas de medicamentos genéricos ou similares contendo este fármaco surgiram no mercado, visando a suplantar a demanda decorrente do aumento no número de prescrições de sinvastatina. Mediante a isso, o objetivo deste trabalho foi validar uma 
Zepon, K.M.; Fratoni, G.; Bernardi, L.S.; Remor, V.T.R. Revista Eletrônica de Farmácia Vol. X (2), $43-57,2013$.

metodologia analítica para doseamento da sinvastatina por espectroscopia de ultravioleta e verificar a equivalência farmacêutica entre três diferentes marcas de venda de comprimidos de sinvastatina $20 \mathrm{mg}$ disponíveis no mercado, avaliando as características físico-químicas através dos ensaios de peso médio, dureza, friabilidade, desintegração, teor de princípio ativo, uniformidade de conteúdo e perfil de dissolução in vitro. 0 método analítico desenvolvido foi validado seguindo parâmetros preconizados pela RE 899/2003, sendo o método considerado preciso, exato, específico, detectável e quantificável, e linear na faixa de concentração de 4 a $12 \mu \mathrm{g} / \mathrm{mL}$ de sinvastatina. Os testes físico-químicos para as três amostras de sinvastatina avaliadas neste trabalho mostram-se de acordo com as especificações descritas na Farmacopeia Brasileira. As eficiências de dissolução das amostras analisadas mostraram resultados satisfatórios, sendo o método utilizado adequado para aplicação em controle de qualidade.

Palavras chaves: Sinvastatina; Estudos de Validação; Controle de qualidade.

\section{ABSTRACT}

Statins such as simvastatin act in the total cholesterol and low density lipoprotein serum decrease. With the patent expiration of simvastatin, new brands in the generic and/or similar medications forms containing this drug appeared on the market, aiming to supplant the demand due to the increase in the number of simvastatin prescriptions. Therefore, the objective of this study was to validate an analytical methodology for simvastatin assay by ultraviolet spectroscopy and determine the pharmaceutical equivalence between three different brands of simvastatin $20 \mathrm{mg}$ tabletsavailable in the market, assessing the physical and chemical characteristics through average weight, hardness, friability, disintegration, active content, uniformity and dissolution profile in vitro. The analytical method was validated following parameters established by RDC 899/2003, and the method considered precise, accurate, specific, detectable and quantifiable, and linear in the concentration range between $4-12 \mathrm{mg} / \mathrm{mL}$ of simvastatin. The physico-chemical tests for the three samples of simvastatin studied in this work showed up in accordance with the specifications described in the Brazilian Pharmacopoeia. The efficiencies of dissolution of the samples showed satisfactory results, the method being suitable for use in quality control.

Keywords: Simvastatin; Validation studies; Quality control.

\section{RESUMÉN}

Las estatinas, como la simvastatina, actúan para disminuir los niveles de colesterol sérico total y de lipoproteínas de baja densidad. Con la expiración de la patente de la simvastatina, nuevas marcas en las formas de medicamentos genéricos y similares aparecieron en el mercado con el objetivo de suplantar la demanda debido al aumento en 
Zepon, K.M.; Fratoni, G.; Bernardi, L.S.; Remor, V.T.R. Revista Eletrônica de Farmácia Vol. X (2), $43-57,2013$.

el número de prescripciones de simvastatina. Por ello, el objetivo de este estudio fue validar una metodología analítica para la determinación de la simvastatina por espectroscopia ultravioleta y determinar la equivalencia farmacéutica entre tres diferentes marcas de venta de simvastatina $20 \mathrm{mg}$ comprimidos a través de peso medio, dureza, friabilidad, desintegración, el contenido activo, uniformidad de contenido y perfil disolución in vitro. El método analítico fue validado siguiendo los parámetros establecidos por la legislación brasileña y el método considerado preciso, exacto, específico, detectables y cuantificables, y lineal en el rango de concentración de 4 y $12 \mathrm{mg} / \mathrm{mL}$ de simvastatina. Las pruebas físico-químicas de las tres muestras de simvastatina estudiadas en este trabajo muestran de acuerdo con las especificaciones descritas en la Farmacopea Brasileña. Las eficiencias de disolución de las muestras mostraron resultados satisfactorios, el método es adecuado para su uso en el control de calidad.

Palabras claves: Simvastatina; Estudios de validación; Control de calidad.

\section{Introdução}

As hiperlipidêmias são alterações metabólicas que ocorrem quando os níveis de lipídeos circulantes estão aumentados na corrente sanguínea, podendo o acúmulo dessas lipoproteínas ricas em colesterol resultar em doenças cardiovasculares e ateroscleróticas. Dados da III Diretrizes Brasileiras sobre Dislipidemias e Prevenção da Aterosclerose associam, à doença aterosclerótica, a principal causa de mortalidade no Brasil, sendo sua prevenção dada pela identificação e controle com o uso de medicamentos antilipêmicos, como as estatinas ${ }^{(1,2)}$.

As estatinas atuam no fígado inibindo a HMG-Coa redutase, enzima fundamental na síntese do colesterol $^{(1)}$. Dentre os fármacos da classe das estatinas, pode-se citar a sinvastatina, fármaco que age na diminuição dos níveis séricos do colesterol total e da lipoproteína de baixa densidade (LDL) em pacientes com hipercolesterolemia, bem como na diminuição dos triglicerídeos e no aumento da lipoproteína de alta densidade $(\mathrm{HDL})^{(3-5)}$.

A resposta terapêutica quanto ao uso das estatinas no tratamento da dislipidemia ocorre entre quatro e seis semanas após início do tratamento, sendo o tratamento por vezes dado de forma crônica. Este cenário, pode representar ao usuário um custo elevado e consequentemente levá-lo a utilizar medicamentos similares ou genéricos como alternativas farmacêuticas de qualidade e menor custo $^{(1,6)}$.Ademais, este cenário também contribui para a inclusão da sinvastatina na Relação Nacional de Medicamentos Essenciais (RENAME) $^{(7)}$.

Assegurar a qualidade dos medicamentos é um atributo de caráter não apenas comercial, mas também, legal, ético e moral. O processo para 
Zepon, K.M.; Fratoni, G.; Bernardi, L.S.; Remor, V.T.R. Revista Eletrônica de Farmácia Vol. X (2), $43-57,2013$.

garantir a qualidade, a segurança e a eficácia dos medicamentos fundamentase no cumprimento da regulamentação sanitária nacional regida pela Agência Nacional de Vigilância Sanitária (ANVISA), a qual tem editado uma série de normas (resoluções e portarias) norteando assim, todo o processo de implementação da política de medicamentos no Brasil( ${ }^{(8-10)}$.

A Lei dos Genéricos (Lei 9.787 de 10 de fevereiro de 1999) estabelece que o genérico seja um medicamento similar e intercambiável a um produto de referência ou inovador. A Resolução da Diretoria Colegiada da ANVISA, trouxe a necessidade de que qualidade, segurança e eficácia dos medicamentos genéricos sejam assegurados. Eles devem ser caracterizados como um equivalente farmacêutico do medicamento de referência através da equivalência farmacêutica (comprovada por ensaios in vitro) e estudos de bioequivalência (comprovada por ensaios in vivo) (11-12).Até 2014, todos os medicamentos similares terão de apresentar os testes de equivalência e bioequivalência, a fim de se garantir que os efeitos são os mesmos dos medicamentos de referência no que tange quantidade e velocidade de absorção do fármaco ${ }^{(12)}$.

Para tanto, o discorrer destes testes de equivalência e bioequivalência requer o cumprimento das normas estabelecidas na legislação vigente e nos compêndios oficiais. Em alguns casos, faz-se necessário primariamente, a validação de uma metodologia analítica antes da execução dos testes, considerando a ausência de métodos em código oficial ou a necessidade de mudanças do método analítico. O teste de validação deverá garantir por meio de estudos experimentais que 0 mesmo atenda às exigências das aplicações analíticas, assegurando a confiança dos resultados. Para tanto deve apresentar especificidade, linearidade, precisão, exatidão e limite de quantificação e determinação, conforme preconiza a RE no 899 de maio de 2003 da ANVISA ${ }^{(14)}$.

Com isso, este trabalho teve por objetivo validar uma metodologia analítica para doseamento da sinvastatina por espectroscopia de ultravioleta, visto não haver na Farmacopeia Brasileira a descrição de uma monografia que permita o doseamento deste fármaco utilizando este método analítico além de avaliar a qualidade dos comprimidos de sinvastatina $20 \mathrm{mg}$ através de análises físico-químicas, doseamento e estudo de perfis de dissolução no intuito de verificar equivalência farmacêutica entre três diferentes marcas comercializadas no Brasil, contemplando o delineamento amostral que envolve o estudo da marca de referência, genérico e similar.

\section{MATERIAIS E MÉTODOS}

Amostras 
Zepon, K.M.; Fratoni, G.; Bernardi, L.S.; Remor, V.T.R. Revista Eletrônica de Farmácia Vol. X (2), $43-57,2013$.

Para a realização do presente estudo foram utilizados comprimidos de sinvastatina $20 \mathrm{mg}$ oriundos de três diferentes indústrias farmacêuticas nacionais, sendo um de referência, um genérico e um similar. As amostras foram nomeadas com $\mathrm{R}$ (para comprimido de referência), G (para comprimidos genéricos) e $S$ (para comprimidos similares), sendo que os comprimidos analisados em cada grupo amostral ( $R, G$ ou $S$ ) pertenciam ao mesmo lote de fabricação.

Substância química de referência

Foi utilizada sinvastatina padrão USP Simvastatin (I0D382), com teor declarado de $99,4 \%$.

\section{Reagentes}

Foram utilizados metanol, para primeira solubilização da sinvastatina padrão e comprimidos, tampão fosfato e o tensoativo lauril sulfato de sódio para os meios de dissolução.

\section{Equipamentos}

Para a realização deste trabalho foram utilizados os seguintes equipamentos: Balança analítica modelo AW 220 (Shimadzu); Aparelho de dissolução modelo VK 7000 e Espectrofotômetro UV/Vis modelo Cary50 BIO ambos da empresa Varian ${ }^{\circledR}$; Durômetro modelo 296/DGP; Friabilômetro modelo300/1; Desintegrador modelo 301/AC, ambos da empresa Nova Ética.

\section{MÉTODOS}

As análises físico-químicas foram realizadas de acordo com os procedimentos descritos na Farmacopeia Brasileira $^{(13)}$.

\section{Peso médio}

A determinação do peso médio foi tomada a partir da análise de 20 comprimidos de cada grupo amostral $(R$, $\mathrm{G}$ e $\mathrm{S})$, sendo pesados individualmente em balança analítica. Utilizaram-se as especificações descritas na Farmacopeia Brasileira $^{(13)}$.

\section{Friabilidade}

Para a realização do teste de friabilidade foram pesados inicialmente 20 comprimidos de cada amostra ( $R$, G e $S)$, sendo em seguida os mesmos submetidos à ação abrasiva gerada por 100 rotações no friabilômetro. Após o teste, os comprimidos intactos e livres de poeira foram novamente pesados. A diferença entre o peso inicial e o final representa a friabilidade medida em função da porcentagem do pó perdido Dureza

O teste de dureza permite determinar a resistência do comprimido ao esmagamento ou a ruptura sob pressão radial. Foram submetidos separadamente, a uma força necessária para esmagá-lo,10 comprimidos de cada amostra ( $R, G$ e $S)$, sendo a dureza 
Zepon, K.M.; Fratoni, G.; Bernardi, L.S.; Remor, V.T.R. Revista Eletrônica de Farmácia Vol. X (2), $43-57,2013$.

mínima aceitável pela Farmacopeia Brasileira $^{(13)}$ igual a $30 \mathrm{~N}$.

\section{Desintegração}

O teste de desintegração permite verificar se os comprimidos de referência, genérico e similar se desintegram dentro do limite de tempo especificado. Esta análise foi realizada a partir de 6 comprimidos de cada amostra ( $R, G$ e $S$ ) utilizando água purificada a $37^{\circ} \mathrm{C} \pm 0,5^{\circ} \mathrm{C}$ como líquido de imersão.

Validação de um método analítico para a quantificação de teor de principio ativo

A validação do ensaio de quantificação foi baseada em critérios estabelecidos pela Resolução RE n० 899 de 29 de maio de 2003 da ANVISA, através da determinação dos parâmetros de linearidade, precisão, exatidão, especificidade, limites de detecção e quantificação(14).

\section{Linearidade}

A linearidade foi determinada através da construção de três curvas de calibração. Para a construção de cada curva de calibração, quantidades apropriadas da solução de estoque de sinvastatina padrão $(1 \mathrm{mg} / \mathrm{ml})$ foram diluídas com lauril sulfato de sódio $0,5 \%$, obtendo-se concentrações de 4, 6, 8, 10 e $12 \mu \mathrm{g} \mathrm{/} \mathrm{mL}$. As amostras foram analisadas no espectrofotômetro de UV no comprimento de onda de $239 \mathrm{~nm}$ utilizando lauril sulfato de sódio 0,5\% como branco. Os dados obtidos permitiram a elaboração de gráficos de concentração das soluções versus absorbância através dos quais as equações das retas e o coeficiente de correlação foram determinados.

\section{Precisão}

O teste de precisão teve por intuito avaliar o grau de dispersão entre a série de medidas obtidas por um mesmo analista (repetibilidade) e entre dois analistas (precisão interanalista). A repetibilidade foi determinada a partir da análise de 6 determinações na concentração de $100 \%$ e a precisão interanalista foi realizada em triplicata com analistas diferentes, para os dois testes foi utilizado uma concentração de $10 \mu \mathrm{g} / \mathrm{mL}$ de sinvastatina.

\section{Exatidão}

A exatidão foi determinada considerando 3 concentrações, baixa $(80 \%)$, média $(100 \%)$ e alta $(120 \%)$ em triplicata, sendo estas concentrações referentes a $8 \mu \mathrm{g} / \mathrm{mL}, 10 \mu \mathrm{g} / \mathrm{mL}$ e 12 $\mu \mathrm{g} / \mathrm{mL}$ respectivamente. Os valores foram expressos pela relação entre a concentração média determinada experimentalmente e a concentração teórica correspondente.

Limite de detecção e quantificação

O limite de detecção foi calculado pelo desvio padrão do intercepto com o eixo " $Y$ " multiplicado por três sob a inclinação da curva de calibração. O 
Zepon, K.M.; Fratoni, G.; Bernardi, L.S.; Remor, V.T.R. Revista Eletrônica de Farmácia Vol. X (2), $43-57,2013$.

limite de quantificação foi estabelecido pelo desvio padrão do intercepto com o eixo "Y" multiplicado por dez, sob a inclinação da curva de calibração. Com isso, se estabelece a menor concentração de fármaco que pode ser quantificada com precisão e exatidão aceitável.

Identificação do teor de princípio ativo dos comprimidos e da uniformidade de doses unitárias

O teor de sinvastatina foi determinado através de espectrofotometria de absorção na região do ultravioleta (UV). Para cada amostra foram pesados 10 comprimidos, sendo em seguida transferidos e triturados em gral até obtenção de um pó fino, deste foi retirado uma quantidade de amostra que equivalesse à média de peso dos comprimidos (obtida a partir da análise de peso médio), sendo esta quantidade dissolvida em um balão volumétrico de $100 \mathrm{~mL}$, utilizando metanol como solvente e submetido ao ultrassom por 15 minutos. Concluída esta etapa, $5 \mathrm{~mL}$ da solução foram filtrados e transferidos para um balão volumétrico de $100 \mathrm{~mL}$ completando-se o volume com solução contendo tampão fosfato e lauril sulfato de sódio $0,5 \%$. A solução padrão de sinvastatina na concentração de 10 $\mu \mathrm{g} / \mathrm{mL}$ foi preparada com auxílio de solução diluente contendo tampão fosfato e lauril sulfato de sódio a 0,5\%. A solução foi então analisada no espectrofotômetro de absorbância na região do UV no comprimento de onda de 239 nm, utilizando solução tampão fosfato e lauril sulfato de sódio 0,5\% para ajuste do zero.

Perfil de Dissolução in vitro

Os perfis de dissolução dos comprimidos de sinvastatina foram realizados de acordo com a metodologia descrita na Farmacopeia Americana(15). Para tanto, foi utilizado aparato II (pá) sob velocidade de agitação de $50 \mathrm{rpm}$ e $900 \mathrm{~mL}$ de solução tampão fosfato $0,01 \mathrm{M}$ $(\mathrm{pH} 7,0)$ e lauril sulfato de sódio $0,5 \%$ em temperatura de $37 \pm 0,5{ }^{\circ} \mathrm{C}$. O estudo foi realizado com seis unidades de cada amostra. Nos tempos préestabelecidos de $5,10,15,20,30$ e 60 minutos alíquotas de $5 \mathrm{~mL}$ foram retiradas e filtradas, sendo o mesmo volume reposto a cuba de dissolução depois de cada coleta. A porcentagem de liberação do fármaco de acordo com o tempo foi determinada através da leitura das absorbâncias em espectrofotômetro UV no comprimento de onda de $239 \mathrm{~nm}$

Análise comparativa dos perfis de dissolução

A avaliação dos perfis de dissolução obtidos deram-se através da análise dos dados por eficiência de dissolução (ED\%), curvas de porcentagem dissolvida versus tempo foram construídas para obter a área sob a curva (ASC) e a área total do gráfico (Equação 1) ${ }^{(14)}$. 
Zepon, K.M.; Fratoni, G.; Bernardi, L.S.; Remor, V.T.R. Revista Eletrônica de Farmácia Vol. X (2), $43-57,2013$.

$$
\left.E D(\%)=\frac{\int_{0}^{T} Y \times d t}{Y_{100} \times T} \times 100 \quad \text { (Equação } 1\right)
$$

Onde $Y$ é a porcentagem de sinvastatina liberada em função do tempo, $T$ o tempo total de ensaio e $Y_{100}$

\section{RESULTADOS E DISCUSSÕES} a quantidade total de fármaco. As médias de eficiência de dissolução obtidas para cada grupo amostral ( $R, G$ e S) foram submetidas à análise de variância (ANOVA), sendo valores de

A Tabela 1 apresenta os resultados referentes a cada grupo amostral ( $R, G$ e $S$ ) frente aos ensaios físico-químicos de peso médio, dureza, friabilidade e desintegração.

$\mathrm{p}<0,05$ considerados estatisticamente significativos.

\begin{tabular}{lllll}
\hline Ensaio & Referência & Genérico & Similar & Conclusão \\
\hline Peso médio (mg) & $206,5 \pm 2,4$ & $202,7 \pm 2,3$ & $208,0 \pm 2,6$ & Aprovado \\
Friabilidade (\%) & 0,01 & 0,10 & 0,02 & Aprovado \\
$\begin{array}{l}\text { Dureza (N) } \\
\begin{array}{l}\text { Desintegração } \\
\text { (min.) }\end{array}\end{array}$ & 93,65 & 88,75 & 87,27 & Aprovado \\
\hline
\end{tabular}

Tabela 1 - Resultados obtidos de peso médio, friabilidade, dureza e desintegração de comprimidos de sinvastatina.

A Farmacopeia Brasileira ${ }^{(13)}$ estabelece que, para os ensaios de peso médio, a variação individual máxima permitida seja inferior a $\pm 7,5 \%$ em relação ao peso médio, não sendo toleradas mais que duas unidades fora do limite especificado. Neste caso, os comprimidos analisados para cada grupo amostral ( $R, G$ e $S$ ) foram considerados aprovados, pois cumprem com os limites estabelecidos neste compêndio oficial.

Os ensaios de friabilidade foram considerados aprovados para cada grupo amostral analisado $(R, G$ e $S)$, pois atendem os limites determinados pela Farmacopeia Brasileira(13), aonde consta que nenhum comprimido pode apresentar-se, ao final do teste, quebrado, lascado, rachado ou partido, devendo apresentar perda de massa igual ou inferior a $1,5 \%$ frente ao seu peso inicial.

Os comprimidos analisados para cada grupo amostral ( $R, G$ e $S$ ) mostraram dureza adequada, já que apresentaram valores de dureza acima do valor mínimo aceitável que é de $30 \mathrm{~N}$ (14). Para os ensaios de desintegração, 
Zepon, K.M.; Fratoni, G.; Bernardi, L.S.; Remor, V.T.R. Revista Eletrônica de Farmácia Vol. X (2), $43-57,2013$.

todos os comprimidos submetidos à análise de cada grupo amostral ( $R, G$, e S) foram considerados aprovados, pois cumpriram com o estabelecido pela Farmacopeia Brasileira(13), ou seja, estavam completamente desintegrados antes de 30 minutos.

Embora a Farmacopeia Americana(15) descreva um método para a quantificação de sinvastatina, o método envolve eluição em gradiente por cromatografia líquida de alta eficiência, sendo este procedimento trabalhoso e demorado. Desta forma, para melhor discorrer com os ensaios relacionados à determinação da qualidade do fármaco contido nos comprimidos de cada grupo amostral analisado, foi necessário primariamente elaborar e validar o método analítico para doseamento da sinvastatina por espectrofotometria UV.

De acordo com a RE 899/2003 ${ }^{(14)}$, para ser considerado válido o método deve apresentar um valor de coeficiente de correlação ( $r$ ) igual ou superior a 0,99 . Deste modo, considera-seo método de doseamento da sinvastatina por UV (Figura 1)validado, tendo em vista que o valor obtido para o coeficiente de correlação (r) foi 0,9994 .

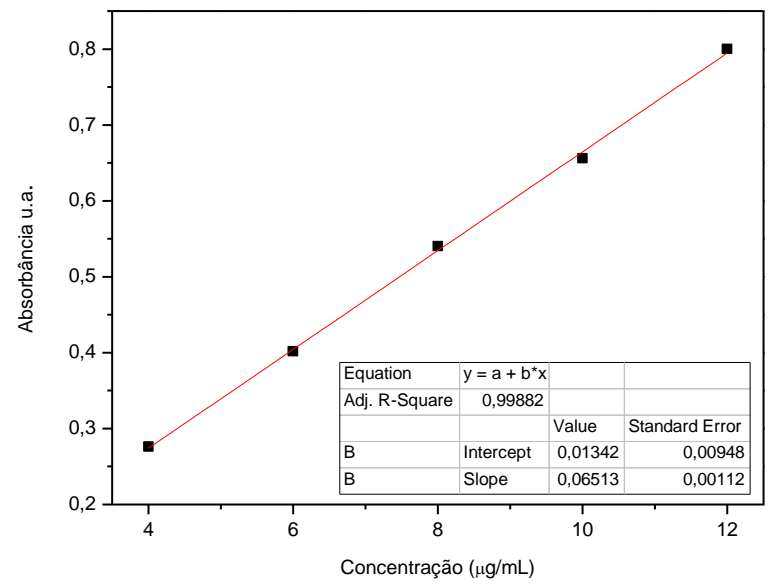

Figura 1 - Representação gráfica da curva de calibração obtida a partir do padrão de sinvastatina.

Os resultados referentes aos repetibilidade estão apresentados na ensaios de precisão interanalista e de Tabela 2.

\begin{tabular}{llllll}
\hline Ensaio & $\begin{array}{l}\text { Conc. } \\
\text { Teórica }\end{array}$ & $\begin{array}{l}\text { Conc. } \\
\text { Média }\end{array}$ & Teor (\%) & DP & DPR (\%) \\
& $10 \mu \mathrm{g} / \mathrm{ml}$ & 9,83 & 98,31 & 0,63 & 0,64 \\
\hline Repetibilidade* & $10 \mu \mathrm{g} / \mathrm{ml}$ & 10,07 & 100.72 & 1,07 & 1,06 \\
Interanalista 1** & $10 \mu \mathrm{g} / \mathrm{ml}$ & 9,75 & 97,55 & 0,85 & 0,87 \\
Interanalista 2** & $10 \mu \mathrm{g} / \mathrm{ml}$ & 10,10 & 101,04 & 0,43 & 0,42 \\
\hline Interanalista 3** & & & &
\end{tabular}

DP: Desvio Padrão. DPR: Desvio Padrão Relativo. 
Zepon, K.M.; Fratoni, G.; Bernardi, L.S.; Remor, V.T.R. Revista Eletrônica de Farmácia Vol. X (2), $43-57,2013$.

* Média de 6 replicatas.

** Média de 3 replicatas.

Tabela 2 - Determinação da precisão interanalista e de repetibilidade.

O valor máximo de desvio padrão encontrado para os diferentes ensaios de precisão foi de $1,07 \%$, estando este valor dentro do limite de $5 \%$ de variação estabelecido pelo Pharmacopoeial Forum $^{(16)}$.

Os resultados obtidos pela análise de limite de detecção, que determina a menor quantidade do fármaco que pode ser detectada pelo método, mostram um valor de concentração de sinvastatina

\begin{tabular}{lll}
\hline Concentração & Teor \% & Média \% \\
\hline & 99,04 & \\
& 99,22 & 99,14 \\
& 99,17 & \\
$10 \mathrm{ug} / \mathrm{ml}$ & 98,98 & 98,96 \\
& 99,21 & \\
& 98,70 & \\
& 99,60 & 99,54 \\
& 99,34 & \\
\hline
\end{tabular}

Tabela 3-Valores de determinação da exatidão.

No que se refere ao teor do princípio ativo, para todos os comprimidos analisados, o teor de sinvastatina (Tabela 4) dispõe-se dentro dos limites especificados pela Farmacopeia igual a 0,56 $\mu \mathrm{g} / \mathrm{mL}$. Para o limite de concentração em que o fármaco pode ser quantificado com precisão e exatidão aceitável, o valor encontrado foi de 1,88 $\mu \mathrm{g} / \mathrm{mL}$.

Conforme mostra a Tabela 3, o método foi exato, uma vez que os valores encontrados ficaram próximos de $100 \%$, em todas as concentrações utilizadas para análise. quantificação, que corresponde à menor

\begin{tabular}{llll}
\hline & Referência & Genérico & Similar \\
\hline Teor $(\mathbf{m g})$ & 10,10 & 9,96 & 9,68 \\
Teor $(\boldsymbol{\%})$ & 101,04 & 99,60 & 96,82 \\
\hline
\end{tabular}

Tabela 4 - Valores obtidos na determinação do teor sinvastatina.

A Farmacopeia Brasileira $^{(13)}$ medicamento que apresentar teor do considera aprovado na primeira etapa do princípio ativo entre $85 \%$ e $115 \%$ e teste de uniformidade de dose, o desvio padrão menor que $6 \%$. Com isso, 
Zepon, K.M.; Fratoni, G.; Bernardi, L.S.; Remor, V.T.R. Revista Eletrônica de Farmácia Vol. X (2), $43-57,2013$.

frente aos resultados apresentados na Tabela 5, foram considerados aprovados em primeiro estágio todos os comprimidos analisados visto que nenhum grupo amostral ( $R, G$ e $S$ ) excedeu o limite estabelecido.

\begin{tabular}{llll}
\hline Produto & Referência & Genérico & Similar \\
\hline $\mathrm{N}$ & Teor $(\%)$ & Teor $(\%)$ & Teor $(\%)$ \\
\hline 01 & 99,25 & 96,52 & 98,96 \\
\hline 02 & 100,20 & 101,56 & 96,34 \\
03 & 101,95 & 102,93 & 100,76 \\
\hline 04 & 96,90 & 100,81 & 96,75 \\
05 & 99,48 & 100,12 & 98,03 \\
\hline 06 & 100,72 & 96,69 & 98,61 \\
\hline 07 & 104,15 & 98,79 & 94,63 \\
\hline 08 & 99,24 & 98,95 & 96,00 \\
\hline 09 & 103,23 & 101,01 & 97,15 \\
\hline 10 & 104,49 & 101,01 & 99,33 \\
\hline Média (\%) & $\mathbf{1 0 0 , 9 6}$ & $\mathbf{9 9 , 8 4}$ & $\mathbf{9 7 , 6 6}$ \\
\hline DP & 2,44 & 2,08 & 1,82 \\
\hline DPR (\%) & 2,42 & 2,09 & 1,87 \\
\hline
\end{tabular}

DP: Desvio Padrão. DPR: Desvio Padrão Relativo.

Tabela 5 -Resultados do teste de uniformidade de dose.

Segundo Dressman (17), os resultados obtidos a partir do teste de dissolução, fornecem informações que possibilitam estabelecer aproximações quanto ao comportamento de liberação in vivo de fármacos a partir de formas farmacêuticas sólidas de uso oral. Sendo assim, a Figura 2 apresenta os perfis de dissolução obtidos a partir dos comprimidos de sinvastatina relativos a cada grupo amostral em estudo ( $R, G$ e S). 
Zepon, K.M.; Fratoni, G.; Bernardi, L.S.; Remor, V.T.R. Revista Eletrônica de Farmácia Vol. X (2), $43-57,2013$.

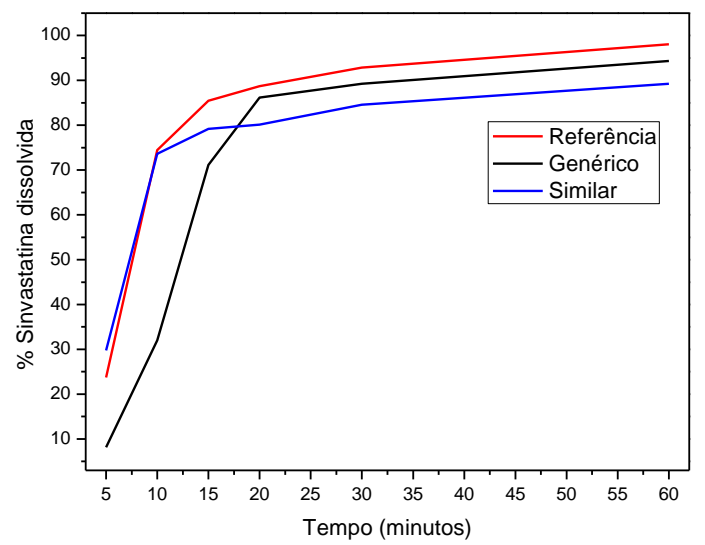

Figura 2 - Perfil de dissolução in vitro dos comprimidos de sinvastatina contendo $20 \mathrm{mg}$.

A Farmacopeia Americana(15) preconiza que para o teste de dissolução, não menos que $80 \%$ da sinvastatina precisam ser liberados em até 30 minutos. Diante disso, como visto na curva de percentagem de sinvastatina dissolvida em função ao tempo, tem-se um comportamento de dissolução semelhante para os diferentes grupos amostrais ( $R, G$ e $S)$, sendo que todas as apresentações liberam mais que $80 \%$ de sinvastatina em até 30 minutos.
A partir dos perfis de dissolução de cada grupo amostral ( $R, G$ e $S$ ) analisado foi possível calcular o parâmetro de eficiência de dissolução. A Eficiência de Dissolução (ED) foi determinada através da razão entre a área sob a curva de dissolução de sinvastatina no intervalo de tempo compreendido entre zero e sessenta minutos (ASC 0-60minutos) e a área total do retângulo $\left(\mathrm{ASC}_{\mathrm{TR}}\right) \mathrm{Na}$ Tabela 6 estão apresentados os valores de eficiência de dissolução.

\begin{tabular}{llll}
\hline $\begin{array}{l}\text { Eficiência de } \\
\text { Dissolução }\end{array}$ & Referência & Genérico & Similar \\
\hline Média ED(\%) & 81,87 & 73,58 & 75,74 \\
DPR (\%) & 1,60 & 1,13 & 1,98 \\
\hline
\end{tabular}

DPR: Desvio Padrão Relativo. ED: Eficiência de dissolução.

Tabela 6- Eficiência de dissolução (\%) dos comprimidos referentes a cada grupo amostral analisado.

A comparação estatística entre as médias de eficiência de dissolução realizada através da análise de variância (ANOVA) indicou haver diferenças estatisticamente significativas $(p<0,05)$ entre os três grupos amostrais ( $R, G$ e $S$ ) analisados quanto à eficiência de dissolução.

\section{Conclusões}


Zepon, K.M.; Fratoni, G.; Bernardi, L.S.; Remor, V.T.R. Revista Eletrônica de Farmácia Vol. X (2), 43 - 57, 2013.

Os testes de qualidade para os comprimidos estudados foram considerados satisfatórios em relação ao peso médio, dureza, friabilidade, desintegração, identificação do teor de princípio ativo e da uniformidade de doses unitárias. Nas condições descritas, a validação do método por espectroscopia de ultravioleta para determinação quantitativa de sinvastatina em comprimidos se mostra de acordo com os parâmetros de validação (linearidade, exatidão, precisão, especificidade e limite de detecção e quantificação) exigidos pela legislação brasileira vigente. O método é, portanto, adequado para análises de rotina de controle de qualidade de comprimidos contendo sinvastatina, visto ser simples, rápido, seguro e de baixo custo. De acordo com os dados estatísticos obtidos, os perfis de dissolução de ambos os produtos genérico e similar seguiram cinética de dissolução em relação o referência. A comparação estatística entre as médias de $E D \%$ indicou diferenças significativas entre os medicamentos de referência, genérico e similar, podendo interferir na intercambialidade entre os mesmos.

\section{Agradecimentos}

Os autores agradecem a Universidade do Sul de Santa Catarina e Universidade Federal de Santa Catarina pelo suporte financeiro.

\section{Referências}

1. MarkmanBEO, Rosa PCP, KoschtschakMRW.Avaliação da qualidade de cápsulas de sinvastatina de farmácias magistrais.Rev. Saúde Públ. 2010; 44: 1055-1062.

2. Giannini SD, Santos RD, Fonseca FH, Moriguchi EH, Maranhão RC, da luz PL, et al. III Diretrizes Brasileiras sobre Dislipidemias e Diretriz de Prevenção da Aterosclerose do Departamento de Aterosclerose da Sociedade Brasileira de Cardiologia.ArqBrasCardiol.2001;77(Suppl 3):1-165.

3. Campo VL, Carvalho I. Estatinas hipolipêmicas e novas tendências terapêuticas. Quím. Nova, 2007; 30: 425-430.

4. DRUGDEX Editorial Staff Simvastatin (Drug Evaluation). In: Klasco, R.K (Ed) DRUGDEX® System, Greenwood Village: Thomson Micromedex, 2007. Available from: http://www.periodicos.capes.gov.br. 
Zepon, K.M.; Fratoni, G.; Bernardi, L.S.; Remor, V.T.R. Revista Eletrônica de Farmácia Vol. X (2), $43-57,2013$.

5. Marina RMR, Emil K, Rita De CBD, Liliete CS. Perfil lipídico e sua relação com fatores de risco para a aterosclerose em crianças e adolescentes. RBAC, 2010; 42:191-195.

6. Monteiro WM, Melo GC, Massunari GK, Hübner DV, Tasca RS. Avaliação da disponibilidade de medicamentos genéricos em farmácias e drogarias de Maringá (PR) e comparação de seus preços com os de referência e similares. Rev. Bras. Cienc. Farm. 2005; 41:333-343.

7. BRASIL. Agência Nacional de Vigilância Sanitária. Portaria $n^{\circ} 533$, de 28 de março de 2012. Estabelece o elenco de medicamentos e insumos da Relação Nacional de Medicamentos Essenciais (RENAME) no âmbito do Sistema Único de Saúde (SUS). Diário Oficial da União, Brasília, DF, 2012.

8. Gil ES, Orlando RM, Serrano SHP, Fisher DCH, Machado SAS, Matias R, Bara MTF, et al. Controle físico-químico de qualidade de medicamentos. 2.ed. São Paulo: Pharmabooks; 2007.

9. Araújo LU, Albuquerque KT, Kato KC, Silveira GS, Maciel NR, Spósito PA, et al. Medicamentos genéricos no Brasil: panorama histórico e legislação. Revista Panam Salud Publica. 2010; 28(6):480-492.

10. BRASIL. Agência Nacional de Vigilância Sanitária. RDC $n^{\circ} 135$, de 29 de maio de 2003. Regulamento técnico para medicamentos genéricos. Diário Oficial da União, Brasília, DF, 2003.

11. BRASIL. Agência Nacional de Vigilância Sanitária. Lei $n^{\circ} 9787$ de 10 de fevereiro de 1999. Lei dos Medicamentos Genéricos. Diário Oficial da União, Brasília, DF, 1999.

12. BRASIL. Agência Nacional de Vigilância Sanitária. RDC $n^{\circ} 134$, de 29 de maio de 2003. Dispõe sobre a adequação dos medicamentos já registrados. Diário Oficial da União, Brasília, DF, 2003.

13. FARMACOPEIA BRASILEIRA, 5a Ed. Brasília: Anvisa, 2010.

14. BRASIL. Agência Nacional de Vigilância Sanitária. RE 899, de 29 de maio de 2003. Guia para a validação de métodos analíticos e bioanalíticos. Diário Oficial da União, Brasília, DF, 2003. 
Zepon, K.M.; Fratoni, G.; Bernardi, L.S.; Remor, V.T.R. Revista Eletrônica de Farmácia Vol. X (2), $43-57,2013$.

15. United States Pharmacopeia. 30. Ed. Rockville: The United States Pharmacopeial Convention, 2007.

16. Pharmacopoeial Forum. The dissolution procedure: development and validation. Pharmacopoeial Previews. 2004; 30(1):351-363.

17. Dressman JB, Amidon GL, Reppas C, Shah VP. Dissolution testing as a prognostic tool for oral drug absorption: Immediate release dosage forms (Review). Pharm. Res. 1998; 15:11-22. 